\title{
Percent Predicted Forced Vital Capacity
}

National Cancer Institute

\section{Source}

National Cancer Institute. Percent Predicted Forced Vital Capacity. NCI Thesaurus. Code C112381.

Forced vital capacity as a proportion of the predicted normal value. (CDISC) 